॥ Gestalten Sie die DGNeurologie mit!

Peter Berlit

Deutsche Gesellschaft für Neurologie (DGN)

Reinhardtstr. 27C, 10117 Berlin, Deutschland

berlit@dgn.org

\title{
DGNeurologie - die neue Zeitschrift unserer Fachgesellschaft
}

\author{
Kompakte, fachliche Informationen auf hohem Niveau und \\ in deutscher Sprache
}

Es tut sich sehr viel bei der DGN (Deutsche Gesellschaft für Neurologie). Von der Öffnung der Fortbildungsakademie auf unserer Jahrestagung für alle Teilnehmenden über die Imagekampagne und die Gründung einer Stiftung beschäftigen uns Fragen der Weiterbildung, der Begleitung neuer diagnostischer und therapeutischer Verfahren und die Auseinandersetzung im Spannungsfeld von Ökonomie und Ethik.

Und jetzt auch noch eine neue Zeitschrift? Muss das wirklich sein? Das werden Sie sich fragen, wenn sie das erste Heft der DGNeurologie in Händen halten. Diese Frage ist natürlich berechtigt. Es gibt eher zu viele deutschsprachige neurologische Zeitschriften, und vieles, von dem was angeboten wird, erscheint redundant. Warum also noch eine Zeitschrift?

Zunächst einmal ist dies eine Zeitschrift unserer Fachgesellschaft - es ist die Zeitschrift der DGN. Wir, Herausgebende sowie Autoren, schreiben für uns, für die DGN. Und Sie, die Lesenden, dürfen von dieser Zeitschrift nicht nur die gewohnten Mitteilungen ihrer Fachgesellschaft, sondern auch fachliche und wissenschaftliche Informationen erwarten - aktuell und auf hohem Niveau.

\section{Herausgeber: Vom Assistenzarzt bis zum Ordinarius}

Ganz bewusst treten wir an diese Aufgabe mit einem jungen und vielseitigen Herausgeberteam heran: Vom Assistenzarzt bis zum Ordinarius und vom niedergelassenen Facharzt bis zu erfahrenen Oberärzten und Wissenschaftlern sind alle Berufssparten und Bereiche der Neurologie im ,editorial board“ vertreten. Neben den Rubriken, die Sie auch in anderen Zeitschriften finden (CME-Weiterbildungsbeiträge und Kasuistiken), überlegten wir uns eine Reihe von besonderen Formaten für Sie, und ich bin gespannt, wie Ihnen diese gefallen.

DGNeurologie 2018 • 1 (1): 1

https://doi.org/10.1007/s42451-018-0012-8

๑) Springer Medizin Verlag GmbH, ein Teil von Springer Nature 2018

\section{Inhalt: Von SOP bis zum Neuro-Quiz}

In jedem Heft wird ein Krankheitsbild grafisch mit seinen essenziellen Bestandteilen im Zoom aufbereitet. Es gibt mit SOP („standard operating procedures“), Arzneimitteltherapiebeiträgen und dem neurologischen Notfall praktische Hilfestellungen für Ihren Klinik- und Praxisalltag. In einer gesonderten Rubrik werden juristische Probleme dargestellt, von Fragen der Arzthaftung bis zu rechtlichen Problemen im Krankenhaus und bei Praxisstrukturen. Natürlich dürfen die bewährten Leitlinien der DGN nicht fehlen, und wir fassen in jedem Heft der DGNeurologie eine aktuelle Leitlinie zusammen. Nicht ganz uneigennützig wählte ich für das erste Heft die Neuauflage der Vaskulitisleitlinie - ein gutes Beispiel für die Interdisziplinarität unseres Fachs.

Internationale, für unsere Berufstätigkeit wichtige Studien werden von erfahrenen Wissenschaftlern für Sie zusammengefasst und kommentiert, und Falldarstellungen werden sich nicht nur dem Besonderen bei häufigen Krankheitsbildern, sondern auch seltenen neurologischen Erkrankungen widmen. Schließlich haben Sie die Möglichkeit, beim Neuro-Quiz ihr Wissen unter Beweis zu stellen. Die Lösung finden Sie jeweils im nächsten Heft der DGNeurologie oder im Mitgliederbereich der DGNHomepage, wo wir Sie jederzeit gerne begrüßen.

Hoffentlich haben Sie bei der Lektüre dieser neuen Zeitschrift so viel Spaß, wie die Herausgebenden und ich beim Zusammenstellen der Beiträge. Für Kritiken und Anregungen aus dem Leserkreis sind wir offen und freuen uns über jede Rückmeldung.

Das erste Heft erscheint rechtzeitig zur Neurowoche in Berlin, bei der wir auch mit dem interaktiven Crossmediaprojekt „reimagine medicine“ auf neue Art und Weise mit Ihnen ins Gespräch kommen wollen. Machen Sie mit! Bei dem Projekt - und bei der Gestaltung unserer Zeitschrift!

Gute Lektüre wünscht Ihnen

Ihr

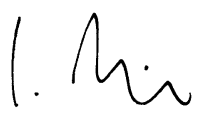

Peter Berlit 